\title{
Nonrelativistic Studies of Diatomic Molecules of Schrödinger Particles with Yukawa Plus Ring-Shaped Potential Model
}

\author{
Akaninyene D. Antia ${ }^{1, ~ *}$, Okon P. Akpan ${ }^{2}$ \\ ${ }^{1}$ Department of Physics, Faculty of Science, University of Uyo, Uyo, Nigeria \\ ${ }^{2}$ Department of Science Technology, Akwa Ibom State Polytechnic, Ikot Ekpene, Nigeria \\ Email address: \\ antiacauchy@yahoo.com (A. D. Antia), akaninyeneantia@uniuyo.edu.ng (A. D. Antia) \\ ${ }^{*}$ Corresponding author
}

\section{To cite this article:}

Akaninyene D. Antia, Okon P. Akpan. Nonrelativistic Studies of Diatomic Molecules of Schrödinger Particles with Yukawa Plus RingShaped Potential Model. Applied and Computational Mathematics. Vol. 6, No. 1, 2017, pp. 54-59. doi: 10.11648/j.acm.20170601.14

Received: November 23, 2016; Accepted: January 21, 2017; Published: February 24, 2017

\begin{abstract}
In this paper, we have solved the Schrödinger wave equation with Yukawa plus ring-shaped potential model using powerful Nikiforov-Uvarov method and obtained the energy eigenvalues and corresponding wave functions in terms of Jacobi and Laguerre polynomials for the angular and radial part respectively. We have also presented the effect of angle dependent solution on radial solutions and also applied our results to obtain numerical values for some selected diatomic molecules which suggest usefulness to other physical systems. We also studied the behavior of our potential graphically for $\mathrm{H}_{2}$ diatomic molecule.
\end{abstract}

Keywords: Noncentral Potential, Diatomic Molecules, Nikiforov-Uvarov Method, Yukawa Potential, Ring-Shaped Potential

\section{Introduction}

Bound state solutions of the Schrodinger equation with physically significant potentials play a major role in quantum mechanics [1, 2]. One of the important tasks in theoretical physics is to obtain exact solution of the physical systems for special potentials (central and noncentral) of interest and some of these potentials are of significant applications in many fields of studies. Noncentral potential in particular has been studied in various fields of nuclear physics and quantum chemistry which could be used for the interaction between deformed pair of nuclei and ring shaped molecules like benzenes [15]. Yukawa potential is one of the short ranged potentials that have been studied in physics, they have Coulombic behavior for small $r$ and are exponentially damped for large $r$, and they have limited number of bound states characterized by the presence of the screening parameter $\alpha$ [6-10]. This model receives great attention for it plays an important role in high energy and particle physics, atomic physics, chemical physics, gravitational plasma physics and solid state physics. In solid state physics and atomic physics, its named the Thomas-Fermi or screened Coulomb potential while in plasma physics it is known as the DebyeHückel potential $[11,12]$.

There has been continuous interest in the solutions of Schrödinger equation, Klein-Gordon and Dirac equations for some noncentral potential. These equations are solved by means of different methods for exactly and approximate solvable potentials. Antia et al solved approximately the Schrödinger equation with HulthenYukawa plus angle dependent potential using NikiforovUvarov (NU) method [13]. Yasuk et al presented an alternative and simple method for the exact solution of the Klein-Gordon equation in the presence of noncental equal scalar and vector potential by using NU method [14]. Hamzavi and Rajabi solved exactly the Dirac Equation with Coulomb plus a novel angel-dependent potential using NU method [15]. They also applied the Schrodinger wave equation to solve the novel angle dependent potential using NU method [16].

The novel angle dependent potential as introduced by 
Zhang and Huang Fu is [17].

$$
V_{\theta}(\theta)=\frac{\gamma+\beta \cos ^{2} \theta+\eta \cos ^{4} \theta}{\cos ^{2} \theta \sin ^{2} \theta}
$$

They solved the Dirac equation for oscillatory potential under a pseudospin symmetry unit. Therefore the motivation of this present work is to solve the Schrödinger equation with Yukawa plus ring-shaped potential model given as

$$
V(r, \theta)=-\frac{V_{0} e^{-\alpha r}}{r}+\frac{\hbar^{2}}{2 \mu}\left(\frac{\gamma+\beta \cos ^{2} \theta+\eta \cos ^{4} \theta}{r^{2} \cos ^{2} \theta \sin ^{2} \theta}\right)
$$

By Taylor's series expansion

$$
e^{-\alpha r}=1-\alpha r+\frac{(\alpha r)^{2}}{2 !}-\frac{(\alpha r)^{3}}{3 !}+\frac{(\alpha r)^{4}}{4 !}+\ldots+\mathrm{O}(\alpha)
$$

Truncating the terms our potential of interest becomes

$$
V(r, \theta)=-\frac{V_{0}}{r}(1-\alpha r)+\frac{\hbar^{2}}{2 \mu} \frac{V_{\theta}(\theta)}{r^{2}}
$$

where $V_{0}$ are the potential depths, $\alpha$ is the screening parameter, $\mu$ is the reduced mass and $\hbar$ is the reduced plank's constant, $\gamma, \beta$ and $\eta$ are arbitrary constants.

We shall obtain the solution of the radial and angle dependent part, present the effect of angle dependent solution on radial solutions and apply our results to some diatomic molecules such as $\mathrm{H}_{2}, \mathrm{CO}, \mathrm{NO}, \mathrm{N}_{2}$ and $\mathrm{Ar}_{2}$.

\section{The Generalized Parametric Nikiforov-Uvarov (NU) Method}

The NU method was presented by Nikiforov and Uvarov [18] and has been employed to solve second order differential equations such as the Schrödinger wave equation (SWE), Klein-Gordon equation (KGE), Dirac equation (DE) etc. The SWE

$$
\psi^{\prime \prime}(r)+[E-V(r)] \psi(r)=0
$$

can be solved by transforming it into a hypergeometric type equation through using the transformation, $s=s(r)$ and its resulting equation is expressed as

$$
\psi^{\prime \prime}(s)+\frac{\bar{\tau}(s)}{\sigma(s)} \psi^{\prime}(s)+\frac{\tilde{\sigma}(s)}{\sigma^{2}(s)} \psi(s)=0,
$$

where $\sigma(s)$ and $\tilde{\sigma}(s)$ must be polynomials of at most second degree and $\bar{\tau}(s)$ is a polynomial with at most first degree and $\psi(s)$ is a function of the hypergeometric type.

The parametric generalization of the NU method is given by the generalized hypergeometirc-type equation as [19]

$$
\psi^{\prime \prime}(s)+\frac{\left(c_{1}-c_{2} s\right)}{s\left(1-c_{3} s\right)} \psi^{\prime}(s)+\frac{1}{s^{2}\left(1-c_{3} s\right)^{2}}\left[-\xi_{1} s^{2}+\xi_{2} s-\xi_{3}\right] \psi(s)=0 .
$$

Equation (7) is solved by comparing it with Eq. (6) and the following polynomials are obtained:

$$
\tilde{\tau}(s)=\left(c_{1}-c_{2} s\right), \sigma(s)=s\left(1-c_{3} s\right), \tilde{\sigma}(s)=-\xi_{1} s^{2}+\xi_{2} s-\xi_{3} .
$$

According to the NU method, the energy eigenvalues equation and eigen functions respectively satisfy the following sets of equation

$$
\begin{gathered}
c_{2} n-(2 n+1) c_{5}+(2 n+1)\left(\sqrt{c_{9}}+c_{3} \sqrt{c_{8}}\right)+n(n-1) c_{3}+c_{7}+2 c_{3} c_{8}+2 \sqrt{c_{8} c_{9}}=0 \\
\psi(s)=N_{n l} s^{c_{12}}\left(1-c_{3} s\right)^{-c_{12}-\frac{c_{13}}{c_{3}}} P_{n}^{\left(c_{10}-1, \frac{c_{11}}{c_{3}}-c_{10}-1\right)}\left(1-2 c_{3} s\right)
\end{gathered}
$$

Where

$$
\begin{aligned}
& c_{4}=\frac{1}{2}\left(1-c_{1}\right), c_{5}=\frac{1}{2}\left(c_{2}-2 c_{3}\right), c_{6}=c_{5}^{2}+\xi_{1}, c_{7}=2 c_{4} c_{5}-\xi_{2}, c_{8}=c_{4}^{2}+\xi_{3}, \\
& c_{9}=c_{3} c_{7}+c_{3}^{2} c_{8}+c_{6}, c_{10}=c_{1}+2 c_{4}+2 \sqrt{c_{8}}, c_{11}=c_{2}-2 c_{5}+2\left(\sqrt{c_{9}}+c_{3} \sqrt{c_{8}}\right) \\
& c_{12}=c_{4}+\sqrt{c_{8}}, c_{13}=c_{5}-\left(\sqrt{c_{9}}+c_{3} \sqrt{c_{8}}\right) \quad \text { (13) } \lim _{c_{3} \rightarrow 0} P_{n}^{\left(c_{10}-1, \frac{c_{11}}{c_{3}}-c_{10}-1\right)}\left(1-2 c_{3} s\right)=L_{n}^{c_{10}-1}\left(c_{11} s\right)
\end{aligned}
$$

In some problem $c_{3}=0$. For this type of problems we And have 


$$
\lim _{c_{3} \rightarrow 0}\left(1-c_{3} s\right)^{-c_{12}-\frac{c_{13}}{c_{3}}}=e^{c_{13} s}
$$

And the solution given in $\operatorname{Eq}(10)$ becomes [20]

$$
\psi(s)=N_{n l} s^{c_{12}} e^{c_{13} s} L_{n}^{c_{10}-1}\left(c_{11} s\right)
$$

where $L_{n}^{\alpha}(s)$ is the Laguerre polynomial.

\section{Factorization Method}

In spherical coordinate the Schrödinger equation with noncentral potential of Eq. (4) can be written as follows [21]

$$
\begin{aligned}
-\frac{\hbar^{2}}{2 \mu} & {\left[\frac{1}{r^{2}} \frac{\partial}{\partial r}\left(r^{2} \frac{\partial}{\partial r}\right)+\frac{1}{r^{2} \sin \theta} \frac{\partial}{\partial \theta}\left(\sin \theta \frac{\partial}{\partial \theta}\right)+\frac{1}{r^{2} \sin ^{2} \theta} \frac{\partial^{2}}{\partial \phi^{2}}\right] } \\
& \times \psi(r, \theta, \phi)+\left(-\frac{V_{0}}{r}(1-\alpha r)+\frac{\hbar^{2}}{2 \mu} \frac{V_{\theta}(\theta)}{r^{2}}\right) \psi(r, \theta, \phi)=E \psi(r, \theta, \phi) .
\end{aligned}
$$

The total wave function in Eq. (17) can be defined as

$$
\psi(r, \theta, \phi)=\frac{R(r)}{r} Y(\theta, \phi)
$$

and by decomposing the spherical wave function in Eq. (17) using Eq. (18) the following equations are obtained:

$$
\frac{d^{2} R(r)}{d r^{2}}+\left[\frac{2 \mu}{\hbar^{2}}\left(E+\frac{V_{0}}{r}(1-\alpha r)\right)-\frac{\lambda}{r^{2}}\right] R(r)=0
$$

$$
\frac{1}{\sin \theta} \frac{\partial}{\partial \theta}\left(\sin \theta \frac{\partial}{\partial \theta} Y(\theta, \phi)\right)+\frac{1}{\sin ^{2} \theta} \frac{\partial^{2}}{\partial \phi^{2}} Y(\theta, \phi)+\left[\lambda-V_{\theta}(\theta)\right] Y(\theta, \phi)=0
$$

where $\lambda=l(l+1)$ and $m^{2}$ are the separation constants. The solution of Eq.(22) is well known [22]. Equations (19) and (21) are the radial and angular parts of Schrödinger equation respectively which are subject for discussion in the preceding section.

\section{Solutions of the Radial Schrödinger Equation}

For eigenvalues and corresponding eigenfunctions of the radial part of the Schrödinger equation from Eq.(19) we have;

$$
\frac{d^{2} R(r)}{d r^{2}}+\left[\frac{2 \mu}{\hbar^{2}}\left(E+\frac{V_{0}}{r}-V_{0} \alpha\right)-\frac{\lambda}{r^{2}}\right] R(r)=0 .
$$

Using the following dimensionless quantities

$$
\varepsilon=\frac{2 \mu E}{\hbar^{2}}, A^{\prime}=\frac{2 \mu V_{o}}{\hbar^{2}}, B^{\prime}=A^{\prime} \alpha
$$

we have

$$
\frac{d^{2} R(s)}{d s^{2}}+\frac{1}{s} \frac{d R(s)}{d s}+\frac{1}{s^{2}}\left[-\left(\varepsilon+B^{\prime}\right) s^{2}+\left(A^{\prime}\right) s-\lambda\right] R(s)=0
$$

Comparing Eq.(25) with Eq.(7) and making use of Eqs.(11-13), the following parameters are obtained:

$$
\begin{gathered}
c_{1}=1 ; \quad c_{2}=c_{3}=0 \\
\xi_{1}=\varepsilon+B^{\prime} ; \quad \xi_{2}=A^{\prime} ; \quad \xi_{3}=\lambda \\
c_{4}=-\frac{1}{2} ; c_{5}=0 ; c_{6}=\varepsilon+B^{\prime} \\
c_{7}=-A^{\prime} ; c_{8}=\lambda+\frac{1}{4} ; c_{9}=\varepsilon+B^{\prime} ; c_{10}=1+2 \sqrt{\lambda+\frac{1}{4}} \\
c_{11}=2 \sqrt{\varepsilon+B^{\prime}} ; c_{12}=-\frac{1}{2}+\sqrt{\lambda+\frac{1}{4}} ; c_{13}=-\sqrt{\varepsilon+B^{\prime}}
\end{gathered}
$$

Substituting Eqs.(26-30) into Eq.(9) and solving the resulting equation explicitly, the energy eigenvalues for the radial part of the Schrödinger equation is obtained as

$$
E_{n l}=V_{0} \alpha-\frac{2 \mu}{\hbar^{2}} \frac{V_{0}^{2}}{\left[(2 n+1)+2 \sqrt{\lambda+\frac{1}{4}}\right]^{2}}
$$

We can immediately obtain the energy eigenvalue of Eq.(31) from hydrogen problem but here we have tried the NU method to show the simplicity of this method. And the corresponding radial wave function is obtained as:

$$
R(r)=N_{n} r^{-\frac{1}{2}+v} e^{-\sqrt{\varepsilon+B^{\prime}} r} L_{n}^{2 v}\left(2 \sqrt{\varepsilon+B^{\prime}} r\right)
$$

where $v=\sqrt{\lambda+\frac{1}{4}}$ and $N_{n}$ is a normalization constant. 


\section{Solutions of the Polar (Angular) Part}

The eigenvalues and the eigenfunctions of the polar part of the Schrödinger equation in this case can be obtained by making use of Eq.(21)

$$
\frac{1}{\sin \theta} \frac{d}{d \theta}\left(\sin \theta \frac{d \Theta}{d \theta} \theta\right)+\left[\lambda-\frac{m^{2}}{\sin ^{2} \theta}-\frac{\gamma+\beta \cos ^{2} \theta+\eta \cos ^{4} \theta}{\cos ^{2} \theta \sin ^{2} \theta}\right] \Theta(\theta)=0
$$

Using the transformation, $q=\cos ^{2} \theta$, Eq. (33) reduces to

$$
\frac{d^{2} \Theta(s)}{d s^{2}}+\frac{(1-3 s)}{2 s(1-s)} \frac{d \Theta(s)}{d s}+\frac{1}{4 s^{2}(1-s)^{2}}\left[-(\lambda+\eta) s^{2}+\left(\lambda-m^{2}-\beta\right) s-\gamma\right] \Theta(s)=0
$$

Comparing Eq.(34) with Eq.(7), the following parameters are obtained

$$
\begin{gathered}
c_{1}=\frac{1}{2} ; c_{2}=\frac{3}{2} ; c_{3}=1 \\
\xi_{1}=\frac{1}{4}(\lambda+\eta) ; \xi_{2}=\frac{1}{4}\left(\lambda-m^{2}-\beta\right) ; \xi_{3}=\frac{\gamma}{4}, \\
c_{4}=\frac{1}{4} ; c_{5}=-\frac{1}{4} ; c_{6}=\frac{1}{16}+\frac{1}{4}(\lambda+\eta) \\
c_{7}=-\frac{1}{8}-\frac{1}{4}\left(\lambda-m^{2}-\beta\right) ; c_{8}=\frac{1}{16}+\frac{\gamma}{4} ; c_{9}=\frac{1}{4}\left(m^{2}+\gamma+\beta+\eta\right) \\
c_{10}=1+\sqrt{\gamma+\frac{1}{4}} ; c_{11}=2+\left(\sqrt{m^{2}+\gamma+\beta+\eta}+\sqrt{\gamma+\frac{1}{4}}\right) c_{12}=\frac{1}{4}+\frac{1}{2} \sqrt{\gamma+\frac{1}{4}} \\
c_{13}=-\frac{1}{4}-\frac{1}{2}\left(\sqrt{m^{2}+\gamma+\beta+\eta}+\sqrt{\gamma+\frac{1}{4}}\right)
\end{gathered}
$$

Substituting Eqs. (35-40) into Eq. (9) gives the relation for $\lambda$ as

$$
\begin{aligned}
\lambda=4(n & \left.+\frac{1}{2}\right)^{2}+2(2 n+1)\left(\sqrt{m^{2}+\gamma+\beta+\eta}+\sqrt{\gamma+\frac{1}{4}}\right) \\
& +2 \sqrt{\left(m^{2}+\gamma+\beta+\eta\right)\left(\gamma+\frac{1}{4}\right)}+m^{2}+2 \gamma+\beta,
\end{aligned}
$$

where $\lambda=l(l+1)$.

The corresponding wave function of the angle dependent part is obtained as

$$
\Theta(s)=N_{m} s^{\frac{1}{4}+\frac{1}{2} \sqrt{\gamma+\frac{1}{4}}}(1-s)^{\frac{1}{2} \sqrt{m^{2}+\gamma+\beta+\eta}} \times P_{n}^{\left(\sqrt{\gamma+\frac{1}{4}}, \sqrt{m^{2}+\gamma+\beta+\eta}\right)}(1-2 s)
$$

Equation (42) can further be written as 


$$
\left.\Theta(\theta)=N_{m}(\cos \theta)^{\frac{1}{2}+\sqrt{\gamma+\frac{1}{4}}}(\sin \theta)^{\sqrt{m^{2}+\gamma+\beta+\eta}} \times P_{n}^{\left(\sqrt{\gamma+\frac{1}{4}}, \sqrt{m^{2}+\gamma+\beta+\eta}\right.}\right)(-\cos 2 \theta)
$$

where $N_{m}$ is a normalization constant.

\section{Effect of Angle Dependent Part on Radial Solutions}

The total energy of the Yukawa plus ring-shaped potential model is obtained by considering the effect of the angle dependent part on the radial part. Substituting Eq.(41) into Eq.(31) yields the energy spectra for this system as

$$
E_{n l m}=V_{0} \alpha-\frac{2 \mu}{\hbar^{2}} \frac{V_{0}^{2}}{[(2 n+1)+2 \delta]^{2}}
$$

Where

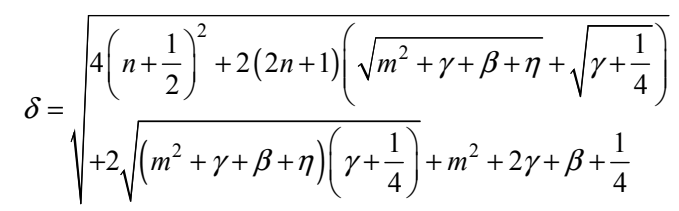

Finally, the total wave function for the system can be written as

$$
\begin{aligned}
\psi(r, \theta, \phi) & =\frac{N_{n m}}{\sqrt{2 \pi}} r^{-\frac{1}{2}+v} e^{i m \phi-\sqrt{\varepsilon+B^{\prime}} r} L_{n}^{2 v}\left(2 \sqrt{\varepsilon+B^{\prime}} r\right) \\
& \times(\cos \theta)^{\frac{1}{2}+\sqrt{\gamma+\frac{1}{4}}}(\sin \theta)^{\sqrt{m^{2}+\gamma+\beta+\eta}} \\
& \times P_{n}^{\left(\sqrt{\gamma+\frac{1}{4}}, \sqrt{m^{2}+\gamma+\beta+\eta}\right)}(-\cos 2 \theta)
\end{aligned}
$$

where $N_{n m}$ is the normalization constant.

\section{Application to Diatomic Molecules}

Diatomic molecular potential are very important to describe the intramolecular and intermolecular interactions and atomic pair correlations in quantum mechanics [23,24]. We shall apply the exact solution of Schrödinger equation for some selected diatomic molecules for the s-wave by setting $V_{0}=D_{e} r_{e}$ in Eq.(44).

$$
E_{n l m}=D_{e} r_{e} \alpha-\frac{2 \mu}{\hbar^{2}} \frac{\left(D_{e} r_{e}\right)^{2}}{[(2 n+1)+2 \delta]^{2}}
$$

where $D_{e}$ is the dissociation energy (interaction energy between two atoms in a molecular system) and $r_{e}$ is the distance of separation between the atoms. Setting $\gamma=\beta=\eta=1$ and making use of Table 1 we obtained numerical values for our total energy eigenvalues.
Table 1. Potential parameters of some selected diatomic molecules [24].

\begin{tabular}{lccc}
\hline $\begin{array}{l}\text { Diatomic } \\
\text { Molecules }\end{array}$ & $\boldsymbol{D}_{\boldsymbol{e}}(\boldsymbol{e V})$ & $\boldsymbol{r}_{\boldsymbol{e}}\left(\begin{array}{l}\boldsymbol{o} \\
\boldsymbol{A}\end{array}\right)$ & $\mu\left(\begin{array}{c}\boldsymbol{o} \\
\boldsymbol{A}\end{array}\right)$ \\
\hline $\mathrm{H}_{2}$ & 4.7446 & 0.7416 & 0.5039 \\
$\mathrm{CO}$ & 10.845 & 1.1282 & 6.8606 \\
$\mathrm{NO}$ & 8.0437 & 1.1508 & 7.4684 \\
$\mathrm{~N}_{2}$ & 11.938 & 1.0940 & 7.0034 \\
$\mathrm{Ar}_{2}$ & 1.6720 & 2.5300 & 53.934 \\
\hline
\end{tabular}

Table 2. Energy eigenvalue for $0.01 \leq \alpha \leq 0.05$.

\begin{tabular}{lllllll}
\hline \multirow{6}{*}{$E_{\text {Elm }}(\mathrm{eV})$} & $\boldsymbol{\alpha}$ & $\boldsymbol{H}_{2}$ & $\boldsymbol{C O}$ & $\boldsymbol{N O}$ & $\boldsymbol{N}_{2}$ & $\boldsymbol{A} \boldsymbol{r}_{2}$ \\
\cline { 2 - 7 } & 0.01 & -6.1310 & -10.183 & -8.8804 & -12.191 & -4.2942 \\
& 0.02 & -5.7632 & -7.3483 & -7.9872 & -8.7922 & -3.6548 \\
& 0.03 & -5.5244 & -6.7375 & -6.8755 & -7.3482 & -3.3075 \\
& 0.04 & -5.0912 & -6.1143 & -5.8349 & -6.5159 & -3.0981 \\
& 0.05 & -4.9396 & -4.9992 & -5.0399 & -6.0141 & -2.9621 \\
\hline
\end{tabular}

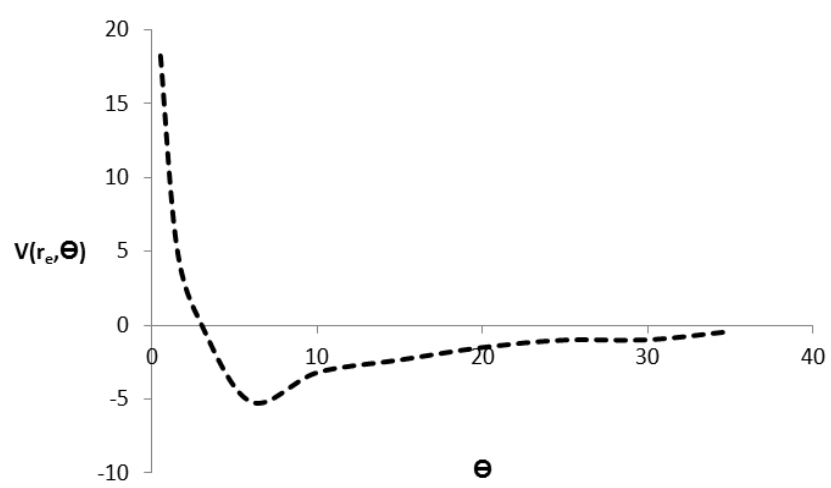

Figure 1. Variation of $V\left(r_{e}, \theta\right)$ with $\theta$ for $\mathrm{H}_{2}$ diatomic molecule.

\section{Conclusion}

The exact bound state solution of Schrödinger wave equation with Yukawa plus ring-shaped potential model using Nikiforov-Uvarov method is obtained and the corresponding wave functions are expressed in terms of Jacobi $P_{n}^{(\alpha, \beta)}(s)$ and Leguerre polynomial $L_{n}^{\alpha}(s)$ for angular and radial solutions respectively. These results are used to study the interactions of the noncentral potential for diatomic molecules. The Yukawa potential is often used to compute bound state normalization and energy levels of neutral atoms while the ring-shaped potentials are used for the interaction between deformed pair of nuclei and ring shaped molecules. The results obtained would have many applications in chemical and molecular physics and the recently reported result of neutron-proton pair in heavy nuclei using perturbation theory [25]. Numerical data for some selected diatomic molecules are presented in Table 2 and Variation of the potential is presented for $\mathrm{H}_{2}$ diatomic molecule in Figure 1. Figure 1 shows that the potential for $\mathrm{H}_{2}$ diatomic molecules decreases downward with increase in $\theta$ and this suggest a good condition for the bound state of this 
system. Also, from the computed results in Table 2, the energy eigenvalues of the selected diatomic molecules decrease with increase in the screening parameter $(\alpha)$. Under limiting cases, our results could be applicable to particles in other physical systems such as Klein-Gordon particles, Dirac particles etc.

\section{References}

[1] Zhang, X. A; Chen, K. and Duan, Z. L. (2005) Chinese physics.14, 42 .

[2] Dong, S. H; Sun, G. H. and Lozada-Cassou, M. (2005) Physical Letter. A328, 299.

[3] Chen. C. Y. and Dong, S. H. (2005) Physical Letter. A335, 374.

[4] Alhaidari, A. D. (2005) Solution of relativistic Dirac-Hulthen problems. Journal of Physics and Mathematics. Gen 38, 3409.

[5] Zhang, M. C. and Wang, Z. B. (2005) Chinese Physical Letter. 22, 2994.

[6] Roy, A. K. (2004) The generalized pseuduspectral approach to the bound state of the Hulthen and Yukawa potentials. Indian Journal of Physics. Vol. 65 No. 1 pp. 1-15.

[7] Yukalov, V; Yukalova, E. and Oliveira, F. (1998). Renormaliziation group solutions for Yukawa potential. Journal of Physics. A31, 4337-4348.

[8] Hamzavi, M; Movahedi, M; Thylwe, K-E and Rajabi, A. (2012) Approximate analytical solution of Yukawa Potential with arbituary angular momenta. Chinese Physics Letter. Vol. 29 No.8.

[9] Hamzavi, M. and IKhdair, S. M. (2013) Relativistic symmetries of fermions in the background of inverse quadratic Yukawa potential as a tensor. Canadian Journal of Physics. A36, 11807-11818.

[10] Ita, B; Ikot, A; Ikeuba, A. and Ebiekpe, V. (2014) Exact solution of Schrödinger equation for the Inverse quadratic Yukawa potential using Nikiforov-Uvarov method. Journal of Theoretical Physics and Cryptography. Vol. 5, pp.7-11.

[11] Alhaidari, A; Bahlouli, H; Nasser, I; and Abdelmonem, M. (2008) an efficient mapped pseudospectral method for weakly bound state. Journal of Chemical Physics. A41, 032001.

[12] IKhdair, S. M. and Sever, R. (2010) Approximate analytical solutions of the general Wood-Saxon potential including the
Spin-orbit coupling term and spin symmetry. Central European Journal of Physics. Vol. 5, 2322-3138.

[13] Antia, A. D; Umo, E. A. and Umoren, C. C. (2015) solution of nonrelativistic Schrödinger equation with Hulthen-Yukawa plus ange dependent potential within the framework of Nikiforov-Uvarov method. Journal of Theoretical Physics and Cryptography. Vol. 10, pp.1-8.

[14] Yasuk, F; Durmus, A. and Boztosun, I. (2006) Exact analytical solution to the relativistic Klien-Gordon equation with a noncentral equal scalar and vector potentials. Journal of Mathematics and Physics. 49, 082302.

[15] Hamzavi, M. and Rajabi, A. A. (2011) Exact solution of Dirac equation with Coulomb plus a novel angle dependent potential. Z. Naturforsch. 66a, 533.

[16] Rajabi, A. A. and Hamzavi, M. (2013) A new coulomb ring shaped potential via generalized parametric Nikiforov-Uvarov method. Journal of Theoretical and Applied Physics. 7:17.

[17] Zhang, M. C and Huang-Fu, G. Q. (2011) Pseudospin symmetry for a new oscillatory ring-shaped noncentral potential. Journal of Mathematics and Physics. 52, 053518.

[18] Nikiforov, A. F. and Uvarov, V. B. (1988) Special Functions of Mathematical Physics. Basel: Birkhauser.

[19] Tezcan, C. and Sever, R. (2009) A general approach of the exact solution of the Schrödinger equation. International Journal of Theoretical Physics. 48, 339.

[20] Antia, A. D. and Ikot, A. N. (2014) Relativistic treatment of Spinless particles Subject to the Hulthen plus Yukawa potential with arbituary $l$-state. Advances in Physics Theory and Applications. Vol. 35, pp.27-35.

[21] Landau, L. D. and Lifshitz, E. M. (1977) Quantum Mechanics. Non-Relativistic Theory. Canada: Pergamon.

[22] Schiff, L. I. (1955) Quantum mechanics. New York: McGraw Hill.

[23] Akcay, H. and Sever, R. (2011) Analytical solutions of schrodinger equation for the diatomic molecular potential with any angular potential. Journal of molecular Structure: Theochem. 845, 133.

[24] Castro, E; Paz, J. and Martin, P. (2006) Journal of molecular Structure: Theochem. 46, 1122.

[25] Khanna, K; Kanyeki, G; Rotich, S; Torongey, P. and Ameka, S. (2010) Anharmonic perturbation of neutron-proton pairs by the paired neutron in heavy finite nuclei. Indian Journal of Pure and Applied Physics. 48, 7-15. 